\title{
470.
}

\section{NOTE ON THE ATTRACTION OF ELLIPSOIDS.}

[From the Monthly Notices of the Royal Astronomical Society, vol. xxIx. (1868-1869), pp. 254-257.]

IF an indefinitely thin shell of uniform density, bounded by two similar and similarly-situated ellipsoids, attracts a point $P$ on its outer surface, it has been shown geometrically by $\mathbf{M}$. Chasles that the attraction is in the direction of the normal at $P$, and is equal to twice the attraction of an infinite plate, the thickness of which is equal to the normal thickness at $P$. Assuming that the attraction is in the direction of the normal, the proof is in fact as follows:- with $P$ as vertex, circumscribe to the interior surface a cone; this divides the shell into three parts; the one, $D+E+F$, exterior to the cone, the other two, $A+B$ and $C$, interior to the cone. It is shown that in the direction of the normal the attraction of $C$ is equal to that of $A+B$;

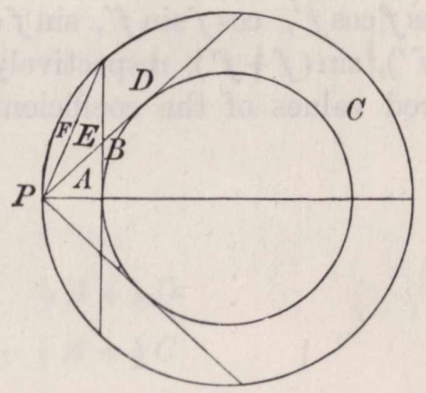

and it is assumed that in comparison with these the attraction of $D+E+F$ may be neglected; the whole attraction is thus equal to twice that of the portion $A+B$. At the point where the normal at $P$ meets the internal surface draw the tangent plane to the internal surface, thus dividing the portion $A+B$ into the solid cone $A$ and 
a remaining portion $B$; it is assumed that in comparison with that of $A$ the attraction of $B$ may be neglected; the whole attraction is thus equal to twice the attraction of the solid cone $A$; and the attraction of this solid cone is in the limit (the aperture or solid angle then becoming $=2 \pi$ ) equal to the attraction of an infinite plate whose thickness is equal to the altitude of the solid cone, that is, to the normal thickness at $P$. And the attraction of the whole ellipsoidal shell is thus ultimately (that is, when the shell is indefinitely thin) equal to twice the attraction of the infinite plate.

It is interesting to ascertain the orders of magnitude of the attractions of the several portions of the shell, which attractions are compared in the foregoing investigation; and this can be done very easily, when, instead of the ellipsoidal shell, we have a spherical shell (bounded by two concentric spherical surfaces). The tangent plane to the inner surface divides the portion $D+E+F$ into two portions $D$ and $E+F$; and if with $P$ as vertex we describe a cone standing on the circle in which the tangent plane meets the outer surface, the last-mentioned portion is hereby divided into the portions $E$ and $F$; the whole shell is thus divided into the portions $A, B, C, D, E, F$, each of them symmetrical in regard to the normal or radius at $P$, and consequently attracting in the direction of this radius. I proceed to find the attractions of each of these portions; it will appear, in accordance with the assumptions of the foregoing investigation, that, taking the radii to be 1 and $1+\alpha$, that is, $\alpha$ the thickness of the shell, and supposing ultimately $\alpha$ to become indefinitely small, the attractions of $A$ and $C$ are each ultimately $=2 \pi \alpha$, that is $=$ to the attraction of the infinite plate, while the attractions of the other portions are of the order $\alpha^{\frac{3}{2}}$, and thus vanish in comparison with that of $A$ or $C$.

The attraction of an indefinitely thin cone or frustum of a cone, length $r$ and solid angle $d \omega$ is $=r d \omega$; considering any such cone having $P$ for its vertex, if the inclination of $r$ to the radius through $P$ is $=\theta$, and if the azimuth of the plane through $r$ and the radius is $=\phi$, then we have $d \omega=\sin \theta d \theta d \phi$, the attraction $r d \omega$ is $=r \sin \theta d \theta d \phi$, and this attraction resolved in the direction of the radius is $=r \sin \theta \cos \theta d \theta d \phi$. For the several cases which have to be considered, the value of $r$ is independent of $\phi$, and the integration in regard to $\phi$ is always from $\phi=0$ to $\phi=2 \pi$; - the attraction is thus in each case $=2 \pi \int r \sin \theta \cos \theta d \theta$, the expression of $r$ in the terms of $\theta$, and the limits of $\theta$ being known for each of the several portions of the shell. Taking $\theta_{1}$ for the semi-angle of the tangent cone, we have it is clear

$$
\sin \theta_{1}=\frac{1}{1+\alpha}, \quad \cos \theta_{1}=\frac{\sqrt{2 \alpha+\alpha^{2}}}{1+\alpha}
$$

and taking $\theta_{2}$ for the semi-angle of the cone which divides the portions $E, F$,

$$
\tan \theta_{2}=\sqrt{2+\alpha}_{\alpha}^{2 \sqrt{2}}, \quad \sin \theta_{2}=\begin{gathered}
\sqrt{2+\alpha} \\
\sqrt{2}(1+\mu)
\end{gathered}, \quad \cos \theta_{2}=\frac{\sqrt{\alpha}}{\sqrt{2(1+\alpha)}} .
$$


For $F$ we have

$$
r=2(1+\alpha) \cos \theta, \quad \theta=\theta_{2} \text { to } \theta=\frac{1}{2} \pi
$$

Integral is

$$
=2(1+\alpha) \int \sin \theta \cos ^{2} \theta d \theta,=\frac{2}{3}(1+\alpha) \cos ^{3} \theta_{2 .} .
$$

For $D+E$ we have

$$
r=2(1+\alpha) \cos \theta, \quad \theta=\theta_{1} \text { to } \theta=\theta_{2,},
$$

Integral is

$$
=2(1+\alpha) \int \sin \theta \cos ^{2} \theta d \theta,=\frac{2}{3}(1+\alpha)\left(\cos ^{3} \theta_{1}-\cos ^{3} \theta_{2}\right)
$$

For $E$ we have

$$
r=\frac{\alpha}{\cos \theta}, \quad \theta=\theta_{1} \text { to } \theta=\theta_{2}
$$

Integral is

$$
=\alpha \int \sin \theta d \theta,=\alpha\left(\cos \theta_{1}-\cos \theta_{2}\right)
$$

For $A$ we have

$$
r=\frac{\alpha}{\cos \theta}, \quad \theta=0 \text { to } \theta=\theta_{1}
$$

Integral is

$$
=\alpha \int \sin \theta d \theta,=\alpha\left(1-\cos \theta_{1}\right)
$$

For $A+B$ we have

Integral is

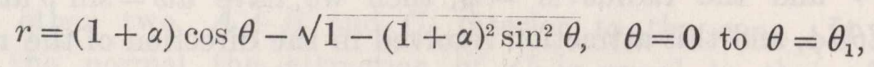

$$
\begin{aligned}
& =\int\left\{(1+\alpha) \cos \theta-\sqrt{1-(1+\alpha)^{2} \sin ^{2} \bar{\theta}}\right\} \sin \theta \cos \theta d \theta \\
& =(1+\alpha)\left(-\frac{1}{3} \cos ^{3} \theta\right)+\frac{1}{3(1+\alpha)^{2}}\left\{1-(1+\alpha)^{2} \sin ^{2} \theta\right\}^{\frac{3}{2}}, \text { between the limits, } \\
& =\frac{1}{3}\left\{(1+\alpha)\left(1-\cos ^{3} \theta_{1}\right)-\frac{1}{(1+\alpha)^{2}}\right\}
\end{aligned}
$$

and subtracting the above value of the integral for $A$, it at once appears that, for $B$, the integral is

$$
=2 \pi\left\{\alpha\left(-1+\cos \theta_{1}\right)+\frac{1}{3}(1+\alpha)\left(1-\cos ^{2} \theta_{1}\right)-\frac{1}{3} \frac{1}{(1+\alpha)^{2}}\right\} .
$$


Hence, calculating the approximate values, and restoring in each case the omitted factor, $2 \pi$, we have

or, if we please,

$$
\begin{array}{clr}
\text { Attraction } & A=2 \pi \alpha-2 \sqrt{ } 2 \pi \alpha^{\frac{3}{2}}, \\
" & B= & \frac{2}{3} \sqrt{2} \pi \alpha^{\frac{3}{2}}, \\
" & C=2 \pi \alpha-\frac{4}{3} \sqrt{2} \pi \alpha^{\frac{3}{2}} \\
" & D= & \frac{4}{3} \sqrt{2} \pi \alpha^{\frac{3}{2}} \\
" & E= & 1 \sqrt{ } 2 \pi \alpha^{\frac{3}{2}} \\
" & F= & \frac{1}{3} \sqrt{2} \pi \alpha^{\frac{3}{2}} ;
\end{array}
$$

$$
\begin{aligned}
& \text { Attraction } \quad A+B=2 \pi \alpha-\frac{4}{3} \sqrt{2} \pi \alpha^{\frac{3}{2}} \text {, } \\
& C=2 \pi \alpha-\frac{4}{3} \sqrt{ } 2 \pi \alpha^{\frac{3}{2}}, \\
& \text { " } D+E+F=\quad \frac{8}{3} \sqrt{ } 2 \pi \alpha^{\frac{3}{2}} \text {; }
\end{aligned}
$$

so that ultimately the attraction of the portion $D+E+F$ vanishes in comparison with those of the portions $A+B$ and $C$; and the attraction of these last, that is, of the whole shell, is $=4 \pi \alpha$, twice the attraction of an infinite plate of the thickness $\alpha$. 